\title{
BoxyBot: a swimming and crawling fish robot controlled by a central pattern generator*
}

\author{
Daisy Lachat, Alessandro Crespi, and Auke Jan Ijspeert \\ School of Computer and Communication Science \\ Swiss Federal Institute of Technology, Lausanne (EPFL) \\ Station 14, CH-1015 Lausanne, Switzerland \\ \{daisy.lachat, alessandro.crespi, auke.ijspeert\}@epfl.ch
}

\begin{abstract}
We present a novel fish robot capable of swimming and crawling. The robot is driven by DC motors and has three actuated fins, with two pectoral fins and one caudal fin. It is loosely inspired from the boxfish. The control architecture of the robot is constructed around a central pattern generator (CPG) implemented as a system of coupled nonlinear oscillators, which, like its biological counterpart, can produce coordinated patterns of rhythmic activity while being modulated by simple control parameters.

Using the CPG model, the robot is capable of performing and switching between a variety of different locomotor behaviors such as swimming forwards, swimming backwards, turning, rolling, moving upwards/downwards, and crawling. These behaviors are triggered and modulated by sensory input provided by light and water sensors. Results are presented demonstrating the agility of the robot, and interesting properties of a CPG-based control approach such as stability of the rhythmic patterns due to limit cycle behavior, and the production of smooth trajectories despite abrupt changes of control parameters.
\end{abstract}

Index Terms-Fish robot, under water robotics, central pattern generator, swimming, crawling.

\section{INTRODUCTION}

The agility and efficiency of animal locomotion tend to fascinate engineers. The skills to coordinate multiple degrees of freedom (DOFs), using compliant actuators (muscles and tendons), and massively parallel control (the central nervous system), give animals an agility and energy efficiency rarely replicated in man-made robots. One of the most impressive features of animals is how they effortlessly deal with multiple redundancies: redundancies in the number of articulated joints, redundancies in the musculature (there are multiple muscles acting on a single joint, and often single muscles acting on multiple joints) and redundancies in muscles (a single muscle is decomposed into multiple motor units). To a large extent, the problem of dealing with these redundancies is solved by central pattern generators, i.e. neural networks capable of producing coordinated patterns of rhythmic activity without any rhythmic inputs from sensory feedback or from higher control centers [1]. Even completely isolated CPGs in a Petri dish can produce patterns of activity, called fictive locomotion, that are very similar to intact locomotion when activated by simple electrical or chemical stimulation [2]. Typically, varying simple stimulation allows modulation of

\footnotetext{
* This work is supported by a Young Professorship grant from the Swiss National Science Foundation to A.J. Ijspeert
}

both the speed and direction of locomotion. From a control point of view, CPGs therefore implement some kind of feedforward controller, i.e. a controller that "knows" which torques need to be rhythmically applied to obtain a given speed of locomotion. Interestingly, CPGs combine notions of stereotypy (steady state locomotion tends to show little variability) and of flexibility (speed, direction and types of gait can continuously be adjusted).

In this article, we apply the concept of CPGs to the control of a novel fish robot. We are interested in testing how a CPG implemented as a system of coupled nonlinear oscillators can be used to control swimming and crawling. Our purpose is to demonstrate that such a system can be a useful basis for producing and modulating a variety of different locomotor behaviors, and for rapidly switching between them. This work follows several related projects on the use of CPGs for controlling a simulated lamprey [3], a simulated salamander [4], [5], and a humanoid robot [6].

In the next sections, we first make a brief overview of related work (Section II). We then present the design of our robot (Section III), and its control architecture (Section IV). Experiments demonstrating different locomotor behaviors are presented in Section V, and our approach is discussed in Section VI.

\section{RELATED WORK}

Multiple fish robots have been designed and realized. Most robots implement anguiliform or carangiform swimming modes, which use mainly the body and the tail to propelled ([7],[8]). Ostraciiform or labriform modes, which use pectoral fins and almost no body motions, have been less studied. Relatively few fish robots are fully autonomous, capable of swimming in 3D and reacting to their environment. For instance, the well-known RoboTuna from MIT, which has been designed to study speed optimization, is attached to a horizontal guide [9].

Several groups are very active in designing autonomous fish robots ([10],[11],[12]). The National Marine Research Institute (NMRI) in Japan, for instance, is working on multiple projects, including maneuvering, swimming performance and modular robotics for water; each robot is built for a particular purpose like up-down motion, high turning performance, or high speed swimming [13]. The University of Essex developed a 3D swimming robotic fish called MT1 which is fully autonomous [14]. A micro 
robotic fish actuated by PZT bimorph actuators has recently been built by the University of California, Berkeley, [15] mimicking a boxfish.

Most of these robots are controlled using traditional control methods that combine (algorithmic) sine-based trajectory generators, and PID feedback controllers. Recently, the concept of CPGs is increasingly used as an alternative approach for online rhythmic trajectory generation [16], [17], [5]. In most cases, the CPGs are implemented as recurrent neural networks or systems of coupled nonlinear oscillators.

To the best of our knowledge, CPGs have rarely been applied to the control of a swimming robot. Arena and Ayers' groups have independently used CPG models inspired by the lamprey locomotor network for controlling tethered lamprey-like robots [18], [19]. The robots were capable of producing traveling waves for propulsion, but autonomous swimming was to the best of our knowledge not explored. In this article, we would like to contribute to underwater robotics in several ways: (1) with the design of a novel fish robot capable of ostraciiform (and labriform) swimming modes and crawling, and (2) with a CPG-based controller that allows agile locomotion in a fully autonomous fish robot.

\section{THE FISH ROBOT BOXYBot}

\section{A. Mechanical design}

The body of the robot is made of two principal parts: the head module, providing two independent joints around the pitch axis (pectoral fins), and the body module, providing a joint around the yaw axis (caudal fin). The modules are rigid cases and are attached together with a rigid part (Figure 1).

The fish robot is designed to implement labriform or ostraciiform swimming modes. Fishes that uses ostraciiform or labriform modes have often inflexible cases for body, like our body and head modules. The caudal fin activated by the body module can be used as a rudder like in labriform mode. Hybrid propulsion (caudal and pectoral) can also be implemented like in ostraciiform mode. However, the concept of the robot is modular and additional modules could easily be added, e.g. to form a body made of a chain of modules. The body module is indeed the same as the one used in an amphibious snake robot that we developed [5].

Casings are molded in polyurethane lightened with phenol microballs. Specific O-rings and grease are used to make the robot waterproof. Total robot's length is $25 \mathrm{~cm}$. The density is slightly higher than that of water and a floater is added to adjust its density to just below 1000 $\left[\mathrm{Kg} / \mathrm{m}^{3}\right]$.

The fins are actuated by 2.83 Watt Faulhaber DC motors and purpose-made gearboxes (reduction factors of 50 and 97.2 for the pectoral and caudal fins). Pectoral fins can make complete rotations, while the motion of the caudal fin is limited to $\pm 60^{\circ}$. The fins are made of 2-mm thick PE plates. The caudal fin has an aspect ratio of 2.9 for 35 $\mathrm{cm}^{2}$, while pectoral fins have 0.6 for $50 \mathrm{~cm}^{2}$. The fins can very easily be changed.

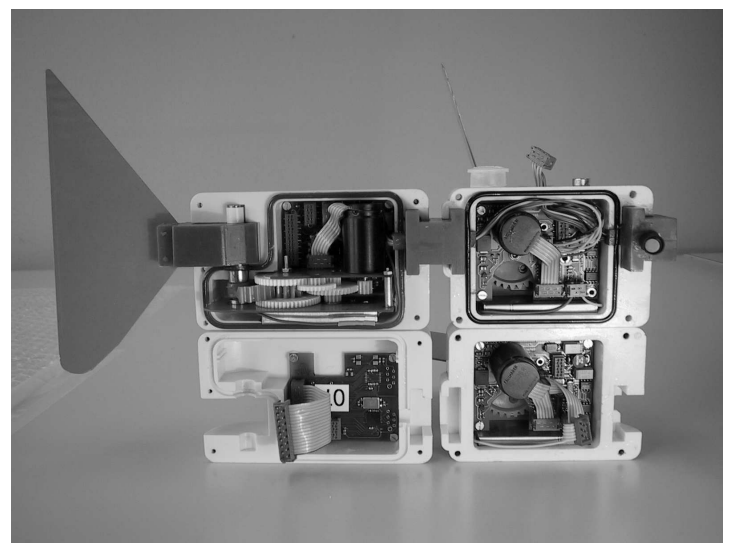

Fig. 1. View from side of the internal components of BoxyBot. The left side of the robot is placed above the right side. On the left is the body module with caudal fin and on the right is the head module with pectoral fins

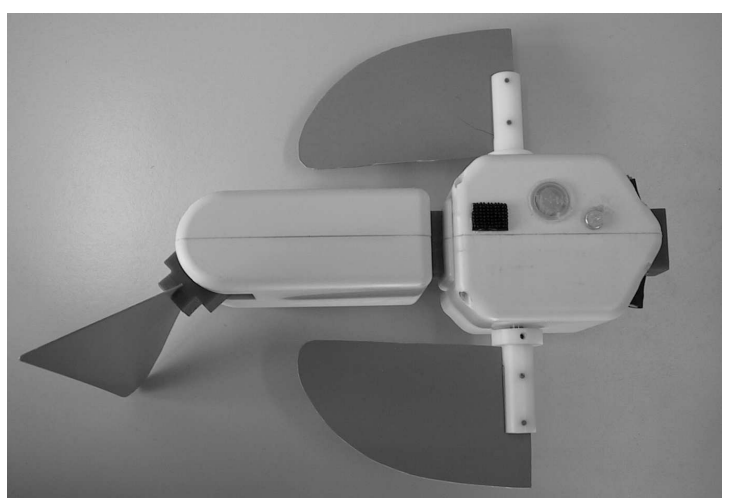

Fig. 2. BoxyBot (view from above)

\section{B. Electronics and sensors}

The robot is fully autonomous. It is controlled by a PIC18F2580 microcontroller at $40 \mathrm{MHz}$ in the head which is used for behavior and locomotion control, with three additional microcontrollers (PIC16F876A) for implementing PD controllers for each motor. The robot locomotion controller (see next Sections) communicates with the motor controllers with an I2C bus and act as the master. The motors are controlled in position using PD controllers. Power is provided by three $4.2 \mathrm{~V} \mathrm{Li}$-Ion batteries (two in the head module and one in the body module).

Light and water sensors are placed in the front of the head in an interchangeable part. The two light sensors are placed in the horizontal plane in transparent polymer tubes making a $60^{\circ}$ angle from one another, a light filter is placed around the tube and can be easily replaced depending on the environment. The water sensor is simply made of two electrical contacts that provide an on signal when the robot is immersed in water (due to water conduction) and an off signal otherwise. A two axis accelerometer (ADXL203) measures accelerations around the roll and pitch axes, but it is not used in the results presented in this article. 


\section{LOCOMOTION CONTROL}

The locomotion controller is composed of a CPG model for producing coordinated oscillations extended by a finite state machine for modulating the CPG and implementing various locomotor behaviors.

\section{A. CPG model}

Our locomotion controller is based on a CPG model implemented as a system of three coupled amplitudecontrolled phase oscillators, one per fin (Figure 3). An oscillator $i$ is implemented as follows:

$$
\begin{aligned}
\dot{\phi}_{i} & =\omega_{i} \\
& +\sum_{j}\left(\alpha_{i j} r_{j} \sin \left(\phi_{j}-\phi_{i}\right)+\beta_{i j} r_{j} \cos \left(\phi_{j}-\phi_{i}\right)\right) \\
\ddot{r}_{i} & =a_{r}\left(b_{r}\left(R_{i}-r_{i}\right)-\dot{r}_{i}\right) \\
\ddot{x_{i}} & =a_{x}\left(b_{x}\left(X_{i}-x_{i}\right)-\dot{x}_{i}\right) \\
\theta_{i} & =x_{i}+r_{i} \cos \left(\phi_{i}\right)
\end{aligned}
$$

where $\theta_{i}$ is the oscillating set-point (in radians) extracted from the oscillator, and $\phi_{i}, r_{i}$, and $x_{i}$ are state variables that encode respectively the phase, the amplitude, and the offset of the oscillations (in radians). The parameters $\omega_{i}, R_{i}$, and $X_{i}$ are control parameters for the desired frequency, amplitude and offset of the oscillations. The parameters $\alpha_{i j}$ and $\beta_{i j}$ are constant coupling weights which determine how oscillator $j$ influences oscillator $i$. The parameters $a_{r}, b_{r}, a_{x}$ and $b_{x}$ are constant positive gains.

The constant parameter values used throughout the article are $a_{r}=20[\mathrm{rad} / \mathrm{s}], b_{r}=5[1 / \mathrm{s}], a_{x}=20[\mathrm{rad} / \mathrm{s}], b_{x}=5$ $[1 / \mathrm{s}]$ and $\alpha_{i j}=0.0[1 / \mathrm{s}], \beta_{i j}=0.0[1 / \mathrm{s}]$ for all $i$ and $j$ except $\alpha_{12}=\alpha_{21}=\alpha_{31}=\alpha_{32}=0.5[1 / \mathrm{s}]$ (oscillators $1,2,3$ respectively correspond to the left-pectoral, right-pectoral, and caudal fins). These values were chosen such that the state variables $r_{i}$ and $x_{i}$ approach $R_{i}$ and $X_{i}$ in a critically damped fashion, and the oscillations of the three fins have a zerophase lag (as long as their intrinsic frequencies $\omega_{i}$ are not too different). The differential equations are solved with Euler integration on the PIC microcontroller with a $7 \mathrm{~ms}$ integration step.

Such a CPG model has several nice properties. The first interesting property is that the system exhibits limit cycle behavior, i.e. oscillations rapidly return to the steadystate oscillations after any transient perturbation of the state variables. The second interesting property is that the control parameters $\omega_{i}, R_{i}$, and $X_{i}$ can be abruptly and/or permanently varied while inducing only smooth modulations of the set-point oscillations (i.e. there are no discontinuities nor jerks). This property will extensively be used in the Results section (Section V) for varying the locomotor behaviors. Another interesting feature is that feedback terms can be added to Equations 1-3 in order to maintain entrainment between control oscillations and mechanical oscillations (however this will not be explored in this article).

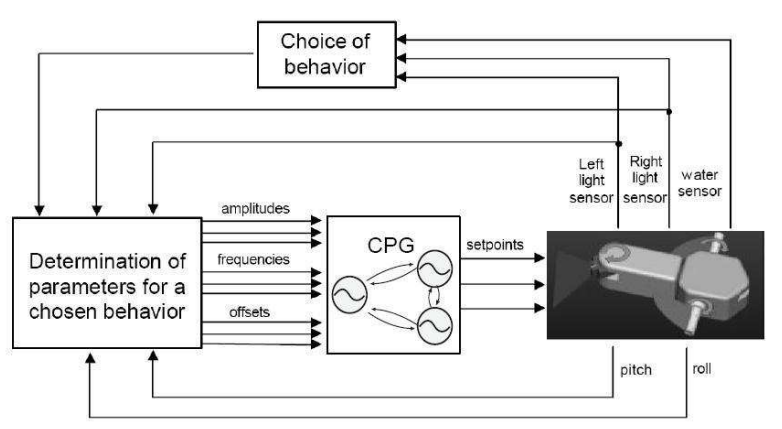

Fig. 3. Diagram of the complete control architecture. While using a predefined behavior the values from light sensors are not used. The values of pitch and roll were not used during the experiments described in this paper.

\section{B. Complete control architecture}

The diagram of the complete control architecture is given in Figure 3. The CPG model produces the set-points $\theta_{i}$ for PD controllers of the three fins. Different locomotor behaviors can be obtained by modulating the CPG control parameters $\omega_{i}, R_{i}$, and $X_{i}$ for the three fins.

Examples of locomotor behaviors include:

- swimming forwards, by oscillating only the caudal fin, both pectoral fins, or all fins.

- swimming backwards, by setting the pectoral offsets ( $X_{1}$ and $X_{2}$ ) to $\pi$ and stopping the oscillations of the caudal fin $\left(R_{3}=0\right)$.

- spinning around the roll axis, by setting the pectoral offsets $X_{1}$ and $X_{2}$ to $\pi / 2$ and $-\pi / 2$.

- turning (around the yaw axis) while swimming, by having a non zero offset $X_{3}$ for the caudal fin.

- turning on the spot, by oscillating the pectoral fins, with one of the pectoral offset to $\pi$.

- swimming up (or down), by setting an offset for both pectoral fins $\left(X_{1}=X_{2}\right)$ between 0 and $\pi / 2(-\pi / 2)$, proportionally to the desired vertical speed.

- crawling, by stopping the oscillations of the fins ( $\left.R_{1}=R_{2}=R_{3}=0\right)$, and applying a continuously increasing offset $\left(X_{1}\right.$ and $\left.X_{2}\right)$ to both pectoral fins. Two possibilities are with $X_{1}=X_{2}$ (both pectoral fins rotate in phase) or $X_{1}=X_{2}+\pi$ (pectoral fins rotate in anti-phase).

For all these behaviors, the speed of locomotion can be varied by adjusting the frequencies $\omega_{i}$ and/or the amplitudes $R_{i}$ of oscillations. Typically the speed of locomotion increases with those parameters until the torque limits of the motors are reached.

We made two types of experiments for testing these different locomotor behaviors. In a first set of experiments, the choice of behavior is done sequentially in a prefixed order without sensory inputs to test the different locomotor behaviors and the transitions between them.

In a second set of experiments, the behavior controller is programmed as a finite state machine to implement a simple phototaxis both in water and on the ground. A 
strong halogen lamp is used as a movable light source and a behavior is chosen on the basis of the values of both light sensors and of the water sensor. The default behavior is to track the light. But if the robot is not in water, it starts to crawl. If the light sensors' signal is too weak, it turns on the spot until it finds the light source again. And if the signals are saturated (i.e. the robot is too close to the lamp), the robot stops.

Once a behavior has been chosen, a second finite state machine determines the 9 control parameters (amplitude, frequency and offset of each motor) to obtain that behavior. For example, if light tracking is chosen, the speed of the robot is controlled inversely proportionally to the amplitude of light by adjusting both the frequency (5) and the amplitude (6) of the oscillations. The caudal offset is controlled proportionally to the difference of light (7).

$$
\begin{aligned}
\omega_{i}=k_{\omega i} \cdot \frac{1}{l_{1}+l_{2}} & i=1,2,3 \\
R_{i}=k_{R i} \cdot \frac{1}{l_{1}+l_{2}} & i=1,2,3 \\
X_{3}=k_{X 3} \cdot\left(l_{1}-l_{2}\right) & X_{1}=0, X_{2}=0
\end{aligned}
$$

where the $k_{i j}$ are gains of the regulator and $l_{1}, l_{2}$ the amplitude of the two light sensors. Note that the CPG never needs any resetting and is continuously running while the 9 control parameters are modified.

\section{RESUlts}

\section{A. Sequentially testing the locomotor behaviors}

We tested the ability of the CPG to produce the different types of locomotor behaviors presented above. Figure 4 presents a sequence of transitions from one behavior to the other. In that sequence, the CPG makes transitions between swimming straight with both pectoral and caudal fins ( $t \leq 2 \mathrm{~s})$, turning with a caudal offset $(2<\mathrm{t} \leq 4 \mathrm{~s})$, swimming straight with only pectoral fins $(4<\mathrm{t} \leq 6 \mathrm{~s})$, swimming backwards $(6<\mathrm{t} \leq 8 \mathrm{~s})$, swimming upwards $(8<\mathrm{t} \leq 10 \mathrm{~s})$, rolling $(10<\mathrm{t} \leq 12 \mathrm{~s})$, slow swimming straight with pectoral and caudal fins $(12<\mathrm{t} \leq 14 \mathrm{~s})$, crawling $(14<\mathrm{t} \leq 18 \mathrm{~s})$, and swimming straight with small amplitudes $(18<\mathrm{t} \leq 20$ s). Figure 5 illustrates forward swimming with pectoral fins. Figure 6 shows the crawling gait using $X_{1}=X_{2}$, it crawls straight forward. If only one pectoral fin is actuated the robot crawls to the left or right. With $X_{1}=X_{2}+\pi$, it crawls forward zigzagging.

Figure 7 shows a turning maneuver by modulating the offset of the tail fin (turn to the right followed by a turn to the left). The minimal radius of turning for this type of turning (with caudal offset) is $0.12 \mathrm{~m}$. Even sharper turns can be made with the turning on the spot maneuver. Movies of the robot can be viewed at http://birg2.epfl.ch/boxybot.

All these transitions are obtained with abrupt changes of the control parameters $\omega_{i}, R_{i}$, and $X_{i}$. Despite these abrupt changes, smooth oscillations are produced by the CPG. Note also that all oscillations remain phase-locked with a zero phase difference thanks to the inter-oscillator couplings.
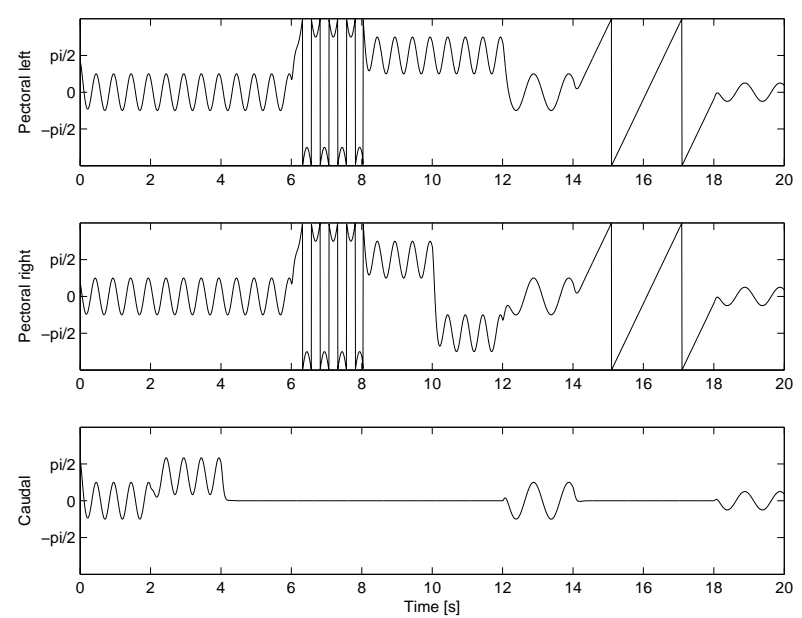

Fig. 4. Sequence of different locomotor behaviors. The graphs show the set-points in radians sent to the three fins. See text for details.
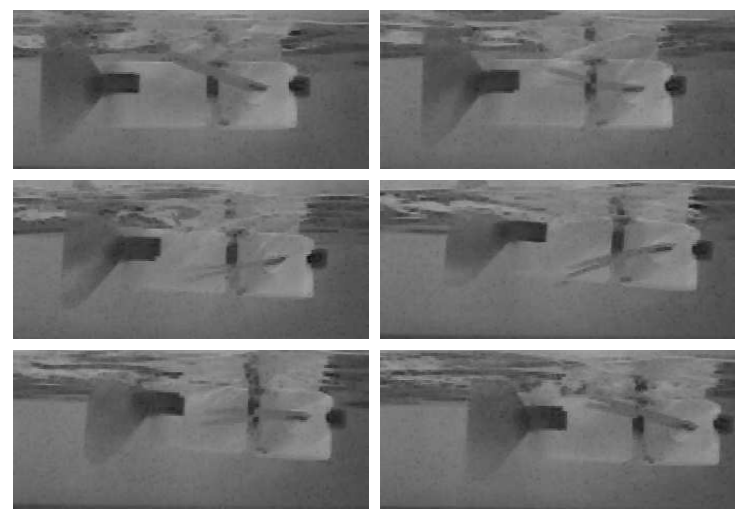

Fig. 5. Snapshots of swimming forward with both pectoral fins (from top left to bottom right).

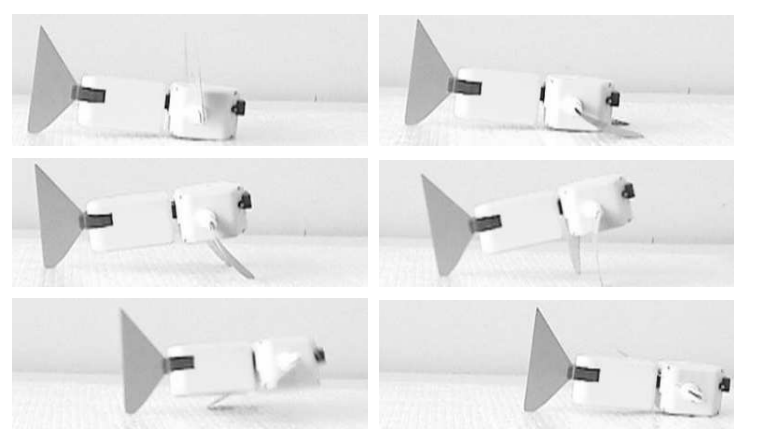

Fig. 6. Snapshots of crawling using continuous rotation of pectoral fins $X_{1}=X_{2}$ (from top left to bottom right).
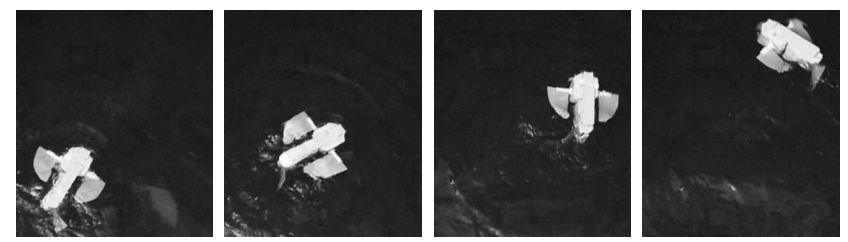

Fig. 7. Snapshots of turning transition. 

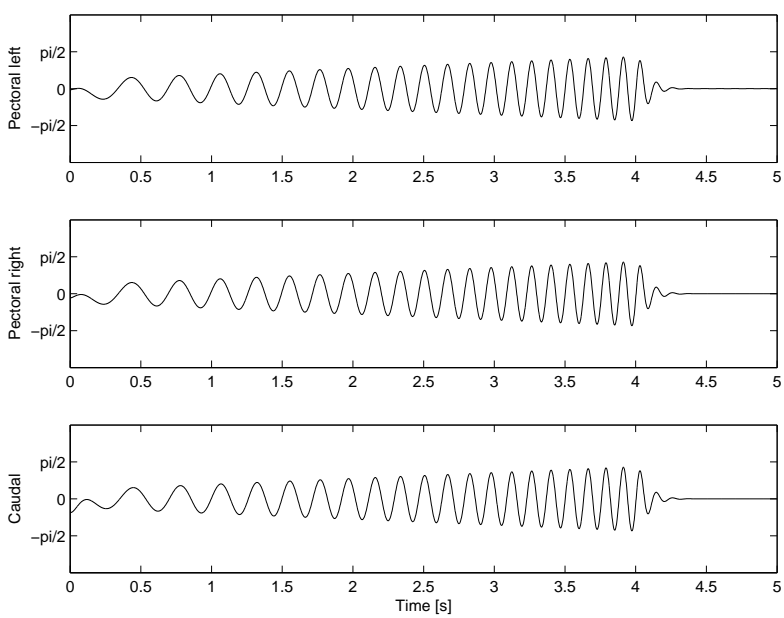

Fig. 8. Acceleration during swimming.
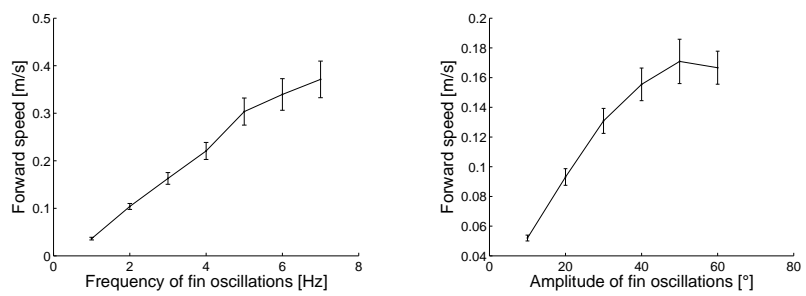

Fig. 9. Variation of forward speed with pectoral fins. On the left, variation with oscillations frequency at a fixed amplitude of $20^{\circ}$. On the right variation with oscillation amplitude at a fixed frequency of $2 \mathrm{~Hz}$. Speed is obtained with the measure of distance covered and time, error bars are calculated from the precision of those two measures.

\section{B. Evaluating the speed of locomotion}

The speed of locomotion can be adjusted by gradually increasing both the frequency and/or amplitude parameters of the CPG. Figure 8 shows the activity of the CPG when both are increased simultaneously.

In order to test how the speed of locomotion depends on the frequency and amplitude of oscillations, we carried out a series of swimming tests. Steady-state speed was measured at different levels of frequencies and amplitudes of all fins. Figure 9 shows the results for variations of frequency at a fixed amplitude (on the left) and for variations of amplitude at a fixed frequency (on the right). As could be expected, the speed of swimming increases with the frequency until the motors reach their torque limits. Similarly, at a fixed frequency, the speed of swimming increases with the amplitude until the oscillations become too large and create braking wakes. Overall, the robot can swim up to 0.37 [m/s] (i.e. 1.4 body lengths per second) at a frequency of $8 \mathrm{~Hz}$ and amplitudes of $\pm 40^{\circ}$ with both pectorals and caudal fins.

\section{Phototaxis}

Using the phototaxis behavior described above, the fish robot is able to reach a static bright light (brighter than the environment) from a maximal distance of $50 \mathrm{~cm}$ and to keep station near the light. It is also able to follow a light that moves slowly (Figure 10). If the light moves too
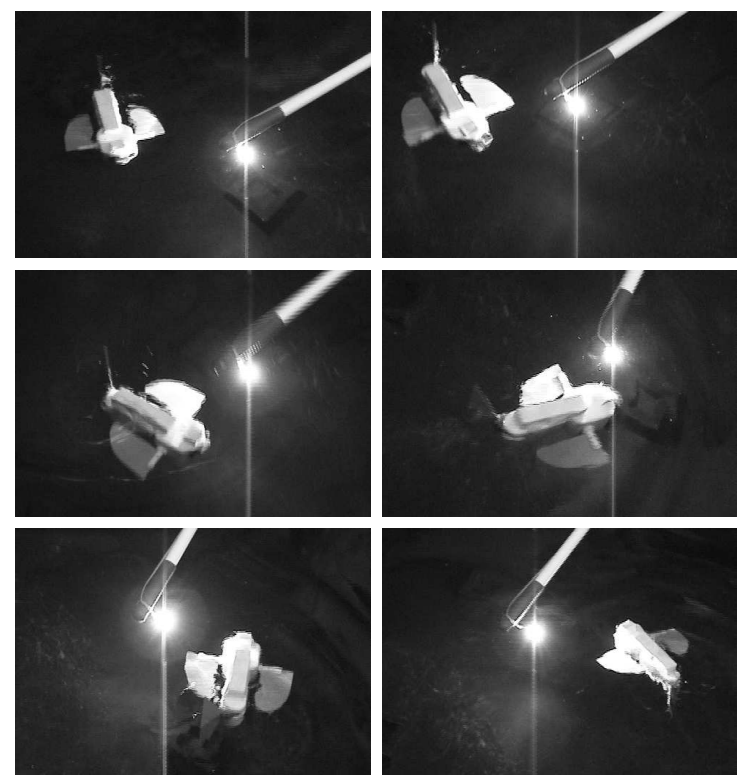

Fig. 10. Snapshots of phototaxis (from top left to bottom right), 2 seconds between each pictures.

quickly on the side, the robot cannot track it because the control law for choosing the speed and caudal offset is very basic (only proportional gains are used). The robot then slowly turns on itself until the light comes into view again, in which case it resumes the light tracking behavior.

\section{Discussion}

BoxyBot has demonstrated its capacity of maneuverability. Using only three fins, it can move in 3D with different types of maneuvers and go out of water using a crawling gait. Finally, the robot can reach a bright light and follow it slowly.

The CPG model proved to be very useful for the online generation of the fin trajectories. It provides the possibility to abruptly change control parameters while ensuring smooth variations of behavior. Producing continuous and smoothly varying set-points is indeed important to limit mechanical damage to the motors and gearboxes, but also to avoid jerks that could destabilize the swimming and crawling gaits. In addition, the phototaxis experiment showed that the CPG model can be continuously modulated and can therefore readily be used by higher level behavior controllers. This is not unlike locomotion control in vertebrate animals where CPGs in the spinal cord produce the rhythmic patterns necessary for locomotion, and higher control centers such as the motor cortex and the cerebellum generate signals for the modulation of speed and direction.

We will extend this work in several directions. First of all, we will explore whether our CPG can be designed to use simpler command signals for initiating and modulating locomotion. In vertebrates, simple tonic (i.e. nonoscillating) signals are sufficient to modulate the speed of locomotion and even to induce gait transitions. In our model, several control parameters need to be changed simultaneously to obtain certain transitions of behavior, and 
we would like to see if this could be simplified. Another point that we intend to explore is whether more complex signal shapes could lead to more efficient swimming. We currently use harmonic (i.e. sine-like) oscillations, and it might be that relaxation-like oscillations (i.e. oscillations that have both a fast and a slow mode) provide faster locomotion for similar frequencies and amplitudes. This will require the use of other types of oscillators in the CPG model. Finally, we would like to explore the integration of sensory feedback in the CPG (not only through modulation of command parameters as done during the phototaxis experiment). In the lamprey, for instance, stretch receptors in the spinal cord ensure that the travelling neural wave remains coordinated with the travelling mechanical wave, and rhythms in the CPG synchronize with externally forced movements of the tail. The CPG model can easily be extended to include similar types of sensory feedback, and we will explore the benefits of such entrainment phenomenon.

\section{ACKNOWLEDGMENT}

We gratefully acknowledge the technical support of André Guignard and André Badertscher in the design and the construction of the robot. We also would like to acknowledge Sacha Constantinescu and Ariane Pasquier for their contribution in designing the light sensors and testing the robot. This work was made possible thanks to the Swiss National Science Foundation (Young Professorship Award to Auke Ijspeert).

\section{REFERENCES}

[1] F. Delcomyn. Neural basis for rhythmic behaviour in animals. Science, 210:492-498, 1980.

[2] S. Grillner. Neural control of vertebrate locomotion - central mechanisms and reflex interaction with special reference to the cat. In W.J.P. Barnes and Gladden M.H., editors, Feedback and motor control in invertebrates and vertebrates, pages 35-56. Croom Helm, 1985.

[3] A.J. Ijspeert, J. Hallam, and D. Willshaw. Evolving swimming controllers for a simulated lamprey with inspiration from neurobiology. Adaptive Behavior, 7(2):151-172, 1999.

[4] A.J. Ijspeert. A connectionist central pattern generator for the aquatic and terrestrial gaits of a simulated salamander. Biological Cybernetics, 85(5):331-348, 2001.

[5] A.J. Ijspeert, A. Crespi, and J.M. Cabelguen. Simulation and robotics studies of salamander locomotion. Applying neurobiological principles to the control of locomotion in robots. Neuroinformatics, 3(3):171-196, 2005.

[6] A.J. Ijspeert, J. Nakanishi, and S. Schaal. Learning attractor landscapes for learning motor primitives. In S. Becker, S. Thrun, and K. Obermayer, editors, Advances in Neural Information Processing Systems 15 (NIPS2002), pages 1547-1554, 2002.

[7] Michael Sfakiotakis, David M. Lane and J. Bruce C. Davies. Review of Fish Swimming Modes for Aquatic Locomotion. IEEE journal of oceanic engineering, vol. 24, no. 2, April 1999.

[8] J.E. Colgate, K.M. Lynch. Mechanics and Control of Swimming: A Review. IEEE journal of oceanic engineering, vol. 24, no. 3, July 2004.

[9] M. S. Triantafyllou and G. S. Triantafyllou. An efficient swimming machine. Sci. Amer., pp. 6470, 1995.

[10] Naomi Kato. Control Performance in the Horizontal Plane of a Fish Robot with Mechanical Pectoral Fins. IEEE journal of oceanic engineering, vol. 25, no. 1, January 2000.

[11] Jindong Liu, Ian Dukes, Rob Knight, Huosheng Hu. Development of fish-like swimming behaviors for an autonomous robotic fish. Department of Computer Science, University of Essex, United Kingdom, 2004.
[12] Junzhi Yu, Min Tan, Shuo Wang and Erkui Chen. Development of a Biomimetic Robotic Fish and Its Control Algorithm. IEEE transactions on systems, man, and cybernetics, Part B: cybernetics, vol. 34, no. 4, August 2004.

[13] Fish Robot Home Page of NMRI. URL: http://www.nmri.go.jp/eng/khirata/fish/index_e.html

[14] Jindong Liu, Ian Dukes and Huosheng Hu. Novel mechatronics design for a robotic fish. IEEE/RSJ International conference on intelligent robots and systems, 2005.

[15] Xinyan Deng and Srinath Avadhanula. Biomimetic Micro Underwater Vehicle with Oscillating Fin Propulsion: System Design and Force Measurement. Robotics and Intelligent Machines Laboratory University of California, Berkeley, USA, 2005.

[16] Y. Fukuoka, H. Kimura, and A.H. Cohen. Adaptive dynamic walking of a quadruped robot on irregular terrain based on biological concepts. The International Journal of Robotics Research, 3-4:187202, 2003.

[17] J. Nakanishi G. Endo, J. Morimoto and G. Cheng. An empirical exploration of a neural oscillator for biped locomotion control. In Proceedings of the 2004 IEEE international Conference on Robotics and Automation (ICRA2004), pages 3036-3042. 2004.

[18] P. Arena. A mechatronic lamprey controlled by analog circuits. In Proceedings 9th IEEE Mediterranean Conference on Control and Automation (MED'01). 2001.

[19] C. Wilbur, W. Vorus, Y. Cao, and S.N. Currie. Neurotechnology for biomimetic robots, chapter A Lamprey-Based Undulatory Vehicle. Bradford/MIT Press, Cambridge London, 2002. 